\title{
Characterising and Exploiting Workloads of Highly Interactive Video-on-Demand
}

\author{
Andrew Brampton - Andrew MacQuire - Michael Fry • Idris A. Rai • \\ Nicholas J. P. Race • Laurent Mathy
}

\begin{abstract}
This paper presents a detailed characterisation of user behaviour for a series of interactive video experiments over a 12 month period, in which we served popular sporting and musical content. In addition to generic VCR-like features, our custom-built Video-on-Demand application provides advanced interactivity features such as bookmarking. The dramatic impact of such functionality on how users consume content is studied and analysed. We discuss in detail how this user behaviour can be exploited by content distributors to improve user experience. Specifically, we study how simple dynamic bookmark placement and interactivity-aware content pre-fetching and replication can reduce the impact of highly interactive media on CDN performance.
\end{abstract}

Keywords Interactive, Video-on-Demand, Sports, World Cup, Content Distribution

\section{Introduction}

In recent years the Internet has increasingly been used to distribute bandwidth-intensive streaming media. Due to the resources required to deliver such content, dedicated Content Distribution Networks (CDNs) are often used to improve the experience of end-users. As such systems evolve, users expect correspondingly improved interactive functionality, something which is increasingly difficult to achieve with diverse content

Andrew Brampton · Andrew MacQuire · Idris A. Rai · Nicholas J. P. Race - Laurent Mathy

Computing Department, Lancaster University, Lancaster, UK

E-mail: \{brampton,macquire,rai,race,laurent\}

@comp.lancs.ac.uk

Michael Fry

University of Sydney, Sydney, Australia

E-mail: michael.fry@usyd.edu.au types exhibiting varied access patterns. In order to provide a high quality of service, modern CDNs must therefore be optimised to respond to user behaviour with regard to different content types.

In this paper, we study user behaviour for an interactive Video-on-Demand ( VoD) system that serves users with a selection of content. Broadly, we served videos in the sporting and musical genres, with respective examples being the entire 2006 FIFA World Cup and 2007 Eurovision Song Contest. A key distinguishing element of our work is that by using our own VoD system, we could offer novel interactive functionality beyond typical VCR-like features (i.e., the ability to pause, resume and skip back and forth within a given video stream). The prime example of this is bookmarking: direct links to points of interest within the video. An example of a bookmark within our sporting content could be a common event such the start of a match, or a potentially more popular event, such as a goal. Likewise, in our musical content, we would typically bookmark the beginning of each distinct piece. Our system also allowed users to contribute their own bookmarks at any time, distinct from those added during the publishing process.

Previous studies making use of entertainment content have witnessed the classic start-to-finish playback model in their access patterns, with occasional VCRlike interactivity. In our experiments, however, user behaviour proved highly dynamic. Users often chose to watch (and replay) small segments of the full video, in a complete departure from the start-to-finish model. The behaviour observed may also exist in other genres with popular highlight (e.g., educational, entertainment, news, etc.). While our scope is limited to sparsely accessed content, we feel our results are of interest and are relevant to significant new genres of on-line video content 
We carried out a statistical analysis of the observed workload resulting from the dynamic user behaviour, and identified models, various metrics and workload properties. These models can be used to drive simulations of the type of interactivity behaviour studied in this paper. We also discuss how delivery networks can exploit the observed behaviour to improve userperceived performance. For instance, we show that the order in which users view bookmarks can be predicted based on previous activity, enabling CDNs to leverage this data for performance gains. We also show how simple dynamic bookmark placement techniques and interactivity-aware content management techniques can improve CDN resource usage and performance.

The remainder of this paper is structured as follows: Section 2 reviews and discusses previous work on VoD workloads. Section 3 describes our experimental setup and methodology. Section 4 then analyses the traces we obtained during our trial period and discusses their significance. Section 5 presents and explores two simple techniques content distribution networks can use to exploit the highly dynamic user behaviour found in our analysis, in order to improve both user satisfaction and resource usage. Finally, Section 6 concludes the paper with a brief summary of results and a discussion of future work.

\section{Related Work}

Much previous work on characterising user interaction has involved authors analysing real-world traces, usually of at least one month in length. A commonlyused repository of such data is the Internet Traffic Archive (ITA) [8]. The ITA maintains a collection of both large and small-scale log files from a number of diverse sources; popular examples being the entire 1998 FIFA World Cup's web server logs, or two months of logs from the NASA Kennedy Space Center in 1995. Unfortunately, many publicly available log files are similarly outdated. Although this may imply that they are likely to be well studied, it equally means they may not be suitable for characterising modern streaming access patterns with VCR-like interactivity. It is perhaps for this reason that many works in this field have instead made use of privately obtained data, often from large networks such as Akamai's [2] or anonymous sources for non-disclosure reasons.

Despite this, many common trends are still observed amongst differing Video-on-Demand workloads, old and new. For instance, it is often found that only a small percentage of objects account for the majority of overall requests. Similarly, a small percentage of the requests often account for a large percentage of the overall data transfer. Accordingly, numerous papers have therefore postulated that the popularity of objects follows a Zipf distribution $[7,13,6,12]$, although some have also indicated that this is not always the case $[5,1]$. In terms of Video-on-Demand session arrivals several suitable (and often heavy-tailed) models have been suggested, e.g., the Poisson, Pareto and Exponential distributions etc. [3]. In general terms, it seems that access patterns depend highly on the nature of the content. Costa et al. highlighted this during their examination of four VoD workloads in three domains (education, entertainment video and entertainment audio). For instance, the authors found that educational content was far more popular in the daytime on weekdays, whereas requests for entertainment-based content were more evenly distributed across the entire week. They also note how a small yet significant fraction of users begin playback at arbitrary positions within the video, and issue an increasing number of requests in correlation with the video length. In terms of the requests issued, 'pause' was found to be, by far, the most common interaction. It was also noted that the probability of a given interaction was dependent on the type of the previous interactions, although the number of these actions was irrelevant [7].

Another common observation was that the popularity of media segments was either roughly uniformly distributed or skewed towards the beginning of videos $[7$, 3]. This is a property of the start-to-finish playback model where users passively watch from the beginning to the end with little interactivity. Following from this, multiple authors have observed that a substantial percentage of media downloads are aborted before completion. Guo et al. suggest that this may be a result of clients conducting "pseudo-streaming"; in other words, simply playing back a downloading video file as it arrives. The authors note that in comparison with real streaming, downloading/pseudo-streaming content is neither bandwidth efficient, nor performance effective $[10,1]$.

The majority of previous work into characterising user behaviour in Video-on-Demand systems has considered simple or VCR-like interactivity exclusively. The impact of newer interactivity features such as bookmarking has not yet been considered to our knowledge. Accordingly, we designed our experiment to allow for the study of such effects, as discussed in the following section. 


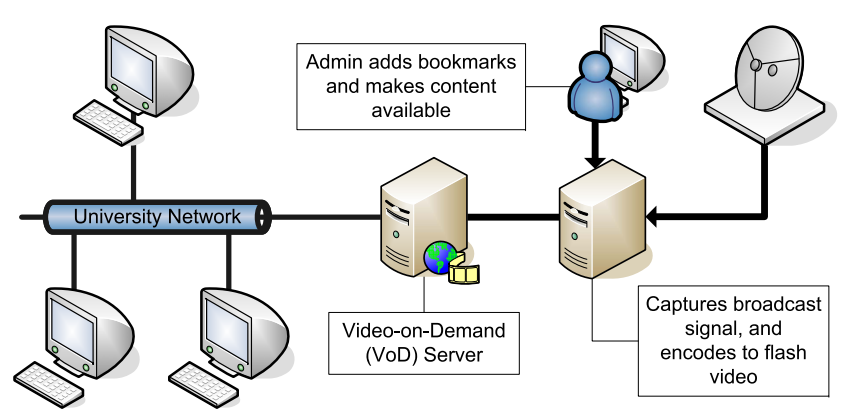

(a) System setup

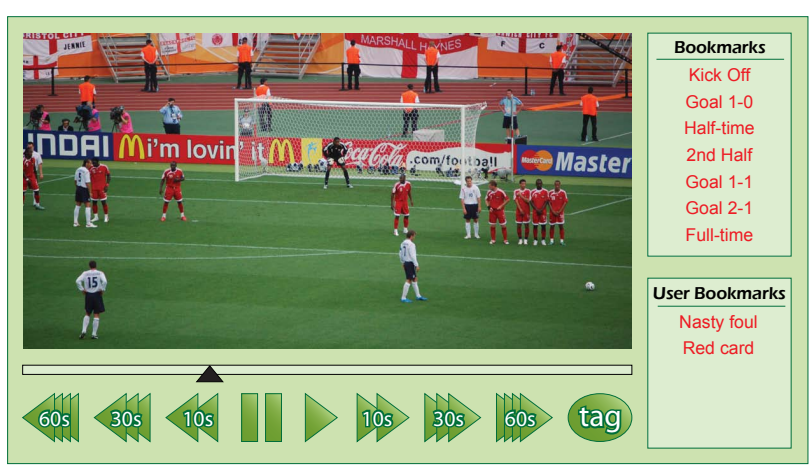

(b) Player interface

Fig. 1: Video-On-Demand system diagrams

\section{Experimental Setup}

We set up a simple, interactive Video-on-Demand system $^{1}$. The system was divided into three main components: the capture server, the Video-on-Demand server, and a web interface as depicted in Figure 1.

Our capture server recorded publicly-broadcast raw MPEG-2 streams of the programmes selected for our experiments. Once this process completed, the system transcoded the stream to high and low bitrate Macromedia Flash 7 FLV files (1 Mbps and 300 Kbps respectively). Administrators would then manually add metadata to the system describing the files as well as marking the location of key events within the videos. These locations are referred to as bookmarks, and typically included events of interest. Within our sport content, for example, goals, fouls and similar occurrences were bookmarked. The final FLV files were then transferred to the VoD server, making them accessible to the users. The full procedure described typically took around twice the length of the recorded video, and so the videos were available shortly after being aired.

The VoD system was an Apache webserver, which served the Flash-based user interface over HTTP. This server was only accessible to staff and students within Lancaster University's campus, and those staff and students connecting remotely via the university's Virtual Private Network (VPN). To aid in logging, all requests made through the user interface were verbose, allowing us to determine exactly which controls users pressed and when. Additionally, each playback window would maintain a periodic (10 second) HTTP-request heartbeat with the server, which was used to determine when connectivity was unexpectedly lost.

\footnotetext{
1 More information about the system and its source code is available at http://www.rcdn.org/
}

To handle user tracking, each user was assigned a unique session ID, which was stored within a HTTP cookie and their URLs. Each event that was logged contained this identifier, allowing us to track individual users throughout their visit to the site. If, however, a user blocked or deleted their cookie, they would appear to be new to the system upon each visit. We note within our analysis where this uncertainty could affect the results.

The web interface consisted of two main sections: an index page allowing the user to select any available video from the system, and the player interface that displayed the video (as shown in Figure 1b). We were aware that the user interface would constrain the users' actions somewhat, and it was therefore designed to be as simple and generic as possible. Forward and backward buttons were provided that allowed seeking 10, 30 and 60 seconds in either direction. As these are relatively short distances, we also provided a seek bar which enabled users to seek to any arbitrarily chosen time. Finally, a list of bookmarks was displayed to the users, which enabled them to jump directly to key events. Bookmarks were added by an administrator, but later the interface was extended to also allow users to submit their own bookmarks (via the tag button), which other users could see and use. User bookmarks often covered events that were not typically bookmarked, but were of particular interest (such as events that came under later scrutiny).

\subsection{Content}

We ran our experiments in two phases, firstly covering the 2006 FIFA World Cup and nine months later a wider range of sport and musical events. The content selections were chosen because they had points of 
interest to bookmark, and would yield sufficient user demand.

The first experiment made available a total of 66 matches from the World Cup (64 from the event itself, and 2 pre-competition friendlies) starting from the $9^{\text {th }}$ of June 2006. Only results after the $13^{\text {th }}$ of June were analysed due to alterations made to the logging system and user interface before that date. Each match was recorded from the beginning of the pre-match commentary through to the end of coverage, and at the very least every goal, penalty, and match start/endpoint (inclusive of half-time) was bookmarked.

From the $13^{\text {th }}$ of April 2007 we began covering new content as part of a second experimental study. This time, our approach was designed to test various autonomic management techniques, and to revalidate our previous experimental results. Furthermore, we wished to determine the relevance of our analysis/models to other genres (such as music). Over the following two months we covered the last six matches from the 2007 UEFA Champions League football tournament, some other miscellaneous football matches, seven Formula 1 races, as well as several recordings from music channels and the 2007 Eurovision Song Contest semi-final and final. The football matches were bookmarked in the same manner as the previous World Cup event. In the Formula 1 content we bookmarked the beginning and end of the race, as well as any noteworthy events such as a driver having to retire (after a crash or technical difficulties). Within the musical content the beginning of each track was bookmarked with its corresponding artist and title. A similar approach was taken with the Eurovision Song Contest, where the beginning of each song was bookmarked with the name of the country taking part.

In total there were 88 videos, each video on average was 2.5 hours in length with a standard deviation of 30 minutes. The maximum video length was 4 hours, and the minimum length 45 minutes. There were 695 bookmarks, with each video having on average 7.8 .

\section{Analysis}

In this section, we use traces from our system to characterise user behaviour and the resulting workload. Using a combination of $R$-Square fitting and KolmogorovSmirnov Tests, models for the various features were determined. Aggregated results are shown when applicable, but in some cases it is more appropriate to show results for individual videos. We noted in many cases the features analysed were similar for each video, so for simplicity we will specifically discuss two individual videos in greater detail: the World Cup's Argentina vs. Serbia and Montenegro match, and the Eurovision Song Contest final. Both were amongst the top 5 most popular videos and were representative of their genres (namely sport and music). We will refer to these files as $\arg -s c g$ and eurovision respectively.

Throughout the two experiments we observed a total of 1800 unique users to the site, with each video receiving on average 68.2 unique users (and an overall maximum of 383). During this period we served 925 hours of video, which equates to 3.3 terabits of data. We received an average demand of $287 \pm 31$ requests per day, with Thursday being the most popular. Throughout the day we saw the typical diurnal sinusoidal access patterns averaging $12 \pm 10$ requests per hour, reaching its peak at midday with an average demand of 29 requests per hour.

We witnessed 123 unique users for $\arg$-scg, and 131 unique users for eurovision, who watched for a combined total of 29.1 hours and 79.6 hours respectively. Note that if an individual does not maintain the same HTTP cookie between sessions (e.g., their cookie is deleted) they will appear as a new unique user. Equally, if two individuals share the same cookie, they will appear as a single unique user. While we expect these cases to be rare, they may however introduce error into the unique user count.

\subsection{Interactions}

Recall that our system allowed various interactive operations, namely pausing, resuming, seeking forwards \& backwards, and jumping to bookmarks. This range of operations, combined with the nature of the content, highly influenced user behaviour. As a result, for most users we observed a complete departure from the typical start-to-finish playback model that has been noted in previous work [7].

Table 1 shows, over the duration of the experiment, the frequency of each action and its corresponding percentage against all other operations. Small forward seeks were used a combined $24.9 \%$ of the time, whereas backward seeking was only used $7.67 \%$. These actions only accounted for the relatively small seeks (10,30, and 60 seconds), whereas potentially large seeks (seek-bar and following bookmarks) made up 34.5\% of all operations. The table also shows that in each session (a viewing of a single video), a user on average used backward actions once, bookmarks and seek bar actions 4.5 times, and forward actions 3.25 times.

Previous studies have shown that the most common action is pause/resume [7], however we see that for our traces, forward operations are by far the most common, 


\begin{tabular}{|c|c|c|c|}
\hline Action & Frequency & Percentage $(\%)$ & Mean \& Standard Deviation $(\sigma)$ per Session \\
\hline Back 10s & 3098 & 4.50 & $0.59(\sigma=3.14)$ \\
Back 30s & 654 & 0.95 & $0.12(\sigma=0.83)$ \\
Back 60s & 1532 & 2.22 & $0.29(\sigma=1.90)$ \\
Forward 10s & 7438 & 10.79 & $1.41(\sigma=8.61)$ \\
Forward 30s & 1804 & 2.62 & $0.34(\sigma=2.93)$ \\
Forward 60s & 7930 & 11.51 & $1.50(\sigma=7.38)$ \\
Seek-bar & 9902 & 14.37 & $1.88(\sigma=7.39)$ \\
Bookmarks & 13857 & 20.11 & $2.62(\sigma=2.63)$ \\
User bookmarks & 1236 & 1.79 & $0.23(\sigma=1.01)$ \\
Pause & 11839 & 17.18 & $2.24(\sigma=7.65)$ \\
Resume & 9616 & 13.96 & $1.82(\sigma=6.80)$ \\
\hline
\end{tabular}

Table 1: Interactions observed throughout the experiment

closely followed by seeking to bookmarks. The table also shows that the number of pause operations account for $17.18 \%$ of all actions. Pausing not being the most common action can be explained by the short session durations observed. This is in accordance with previous work which found a positive correlation between session time and the number of pause operations [14].

To better understand how users navigated through a bookmarked video, we analysed the behaviour in the arg-scg and eurovision videos, which had 10 and 24 bookmarks respectively. In Figures $2 \mathrm{a} \& 2 \mathrm{~b}$ each point is a seek that is identified by a "from" time on the $\mathrm{x}$-axis and a "to" time on the y-axis. A point $x, y$ therefore represents a user that has jumped from their current playback point $x$ to a new point, $y$. Vertical and horizontal lines in the figures denote the position of the bookmarks. The diagonal line is a current-time marker such that seeks forward are points which lie above it, while seeks backward appear below it. Therefore, no point can fall precisely on the diagonal. It is immediately obvious from the figures that many points are on horizontal lines, implying that most seeks were to the bookmarks.

The forward seek buttons appear to have been mostly used for skipping to the next event, shown on both figures as points slightly above the diagonal line between the bookmarks. This could be due to user unfamiliarity with the bookmark interface, or possibly users simply browsing the video. Backward actions were typically used around bookmarks, where users would often re-watch the bookmarked event. In some cases users may also have wished to see video immediately preceding the bookmark. An example of this is shown in Figure 2 a before the bookmark at time 2815, where users sought up to 75 seconds backwards to see more of the build up to the goal.

Clusters of points can also be seen on horizontal lines shortly after a vertical line, indicating that users jumped from bookmark to bookmark. In fact, the concentration of clusters of point just above the diagonal time reference indicates that users have a tendency to follow bookmarks in sequence, as exemplified in Figure $2 \mathrm{~b}$.

Overall, for both videos these results demonstrate that users did not simply view continuously start-tofinish, and were in fact highly influenced when presented with bookmarks.

\subsection{Seek Distance}

The understanding of locality is important for caching and pre-fetching algorithms. By looking at how far users sought we can determine the probability of accessing media nearby the playback point. We therefore define seek distance as the absolute difference, in seconds, between a user's current playback point and their requested seek destination.

Figures 3a \& 3b display a CDF of seek distance for backward and forward actions. A large proportion of seeks (between 50\%-70\%) are of a 15 seconds, 30 seconds, or 60 seconds values. These seeks represent the short seek button presses. $40 \%$ of backward seeks were less than or equal to 15 seconds in length. This property could be exploited by keeping a small client side buffer of previously watched segments, which would satisfy many backward seeks if the user has already viewed them.

Even though small seeks are the majority, there are between $30 \%$ and $50 \%$ of seeks which are further than 60 seconds. These seeks consist of jumps to bookmarks or "blind" seeks with the seekbar. These long range seeks are log-normally distributed with a mean of 1968 seconds and 1630 seconds for forward and backward seeks respectively. They can be fitted to log-normal models with parameters $\mu=6.8269$ and $\sigma=1.5953$ for forward seeks, and $\mu=6.3273$ and $\sigma=1.7906$ for backward seeks. It can been seen that the backward distribution has a greater positive skew than the forward distribution, thus it will generate many small seeks. 


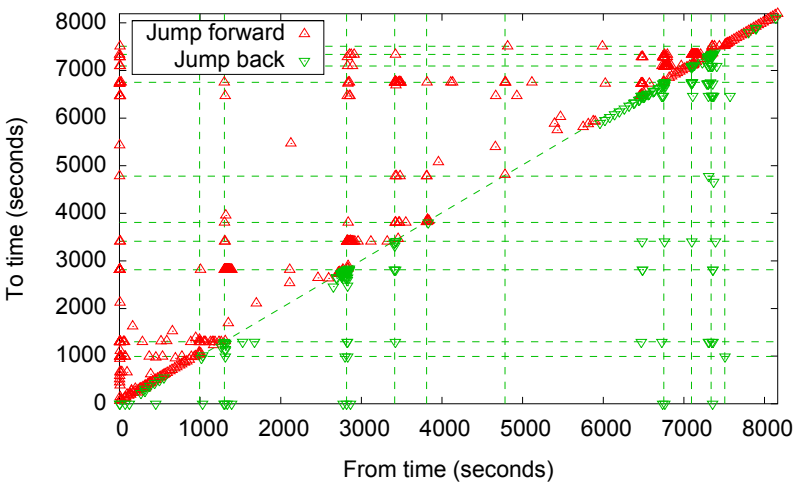

(a) Argentina vs. Serbia and Montenegro

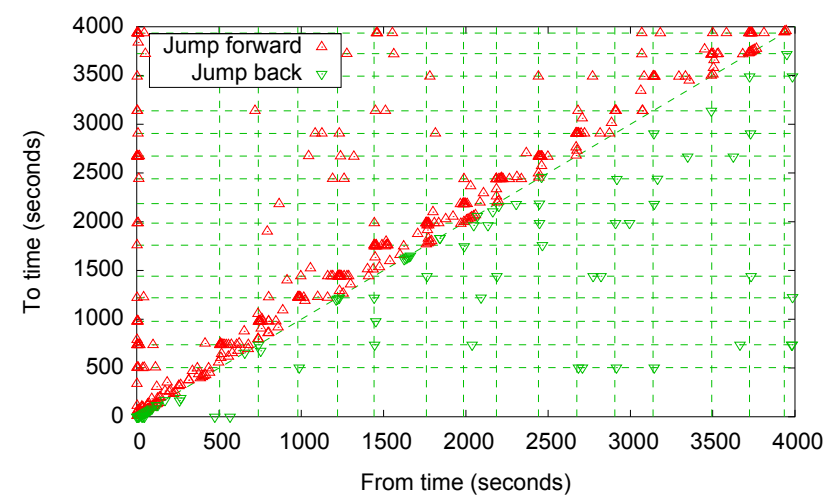

(b) Eurovision (cropped at 4000 seconds for clarity)

Fig. 2: Jumps made by users within two videos

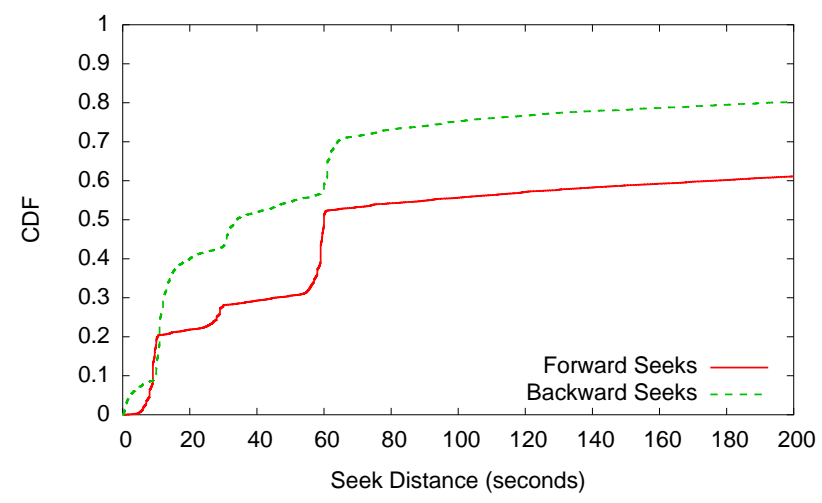

(a) Small scale

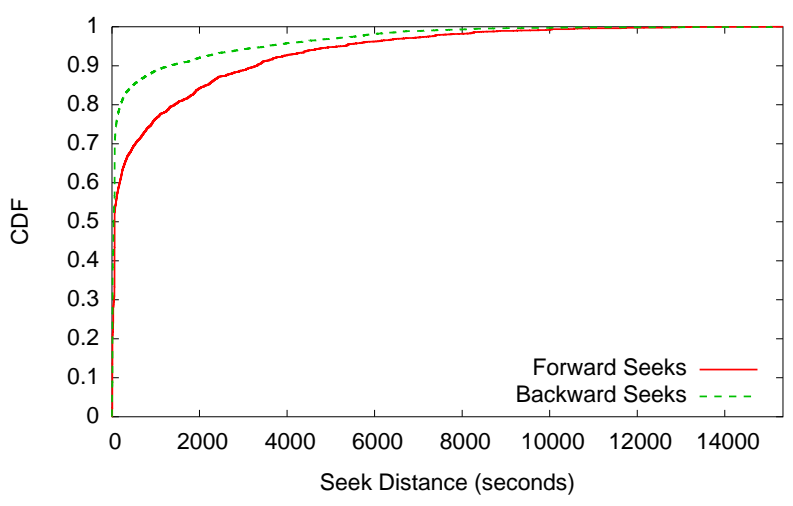

(b) Large scale

Fig. 3: $C D F$ of seek distance

These behaviours exhibit a high degree of spatial locality, with the majority of seeks being within 60 seconds. Regarding long-ranged seeks, the log-normally distributed models imply that some very large distance seeks do occur, but the majority of seeks are shorter. Overall the seek distances exhibit a median of $60 \mathrm{sec}-$ onds for forward seeks and 34 seconds for backward seeks.

\subsection{Popularity}

We study popularity in terms of the number of viewers who watched an object or a segment. An object in our system is a single video whereas a segment is a section of video one second in length.

The ranking for both object and segment popularity is shown in Figure 4. The eurovision, and arg-scg were approximately 10,000 seconds in length, causing 10,000 segments to be ranked for each video. Recall that only
88 videos were available, so the lowest object rank is 88 . Our analysis reveals that object popularity does not follow the typical power-law distribution observed within CDNs $[6,3,15]$ but instead is a normal distribution with parameters $\mu=60$ and $\sigma=32$. This can be attributed to the nature of our videos and the relatively few new objects each day.

The popularity of one-second segments for all the videos exhibit a Weibull distribution with parameters $\lambda=2.887$ and $k=0.69527$. Log-normal distributions provide the best fits for the arg-scg and eurovision results independently with parameters $\mu=2.00, \sigma=$ 0.587 and $\mu=2.32, \sigma=0.567$ respectively. Note that log-normal and Weibull distributions closely relate to power-law or heavy-tailed distributions $[11,9]$ : they are skewed distributions where a small percentage of samples contributes to a sizeable weight of their distribution. We observe that a small percentage, (the 10\% most popular segments), accounted for about $44 \%$ of all requests. Previously, Costa et al. [7] found that for ed- 


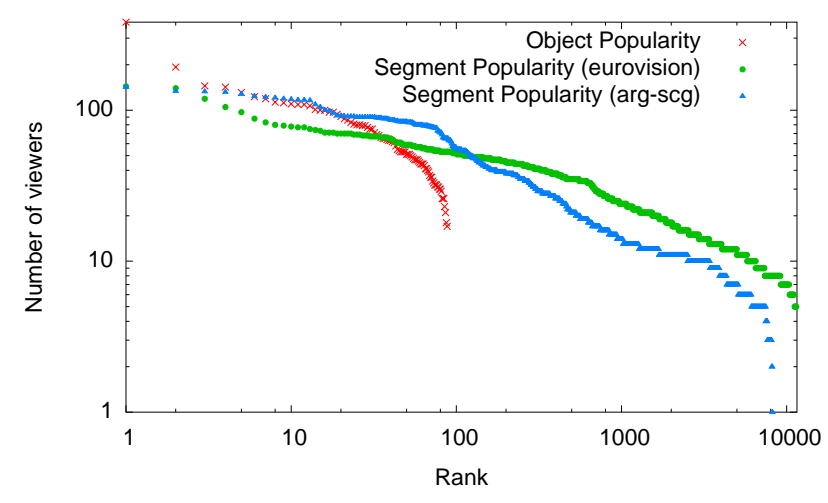

Fig. 4: Object and segment popularity

ucational and entertainment content, the popularity of segments is roughly uniformly distributed with a slight skew towards the beginning for entertainment content. Our result, however, implies that there are segments with orders of magnitude more viewers than others.

To illustrate the order-of-magnitude differences in viewers, we present Figures $5 \mathrm{a} \& 5 \mathrm{~b}$ which show the popularity of each second of video for $\arg -s c g$ and $e u$ rovision respectively. The vertical lines signify the position of the bookmarks; note for the eurovision video there were no bookmarks after 6000 seconds since the performances bookmarked were only in the first half. It is clear from the figures that there are peaks of popularity, highly influenced by the bookmarks. In arg-scg (and in other sport content) we observe that most of the bookmarks are equally popular. However, in the eurovision (and other music genres), we observe there is a greater variance in the popularity of the bookmarks. This can be attributed to sports having numerous events which all users wish to watch, however in music videos there may be only certain artists which interest the user.

Popularity metrics are important to many CDN algorithms as they help to decide which resources to allocate to each object. We have seen that bookmarked videos provide a content format with specific segments of interest (goals, for example). This result emphasises the use of partial caching techniques [4] to cache only popular segments.

\subsection{Longevity}

The popularity of both videos and bookmarks in our system faded over time. We call the duration at which any such item remains utilised its longevity. The study of a video or bookmark's longevity can aid cache re- placement policies, as well as other content management decisions.

Figure 6 shows the popularity of all our bookmarks versus the time they were first used. The figure suggests that following an initial peak and a slight resurgence, there was a rapid decrease in interest after a short period. R-Square fitting reveals that the bookmark longevity can be suitably estimated using a Weibull distribution with $\lambda=3.10$ and $k=0.615$. This suggests that the popularity exhibits long-tailed properties. We also observe that $40 \%$ of the bookmark usage occurs within 24 hours, with the remainder slowly occurring over the following weeks.

The popularity of videos decreased over time, but this is not true for the popularity of segments within the videos. The segments which were popular when the video was first published were still popular within the video weeks later, long after the video had lost popularity. This was tested on each video by calculating the distribution of segment popularity for the first $50 \%$ of requests versus the last $50 \%$ of requests. The difference in distributions was minor, with an average R-Square value of 0.9 . On a visual inspection of the number of viewers per second, it was clear that the popularity still focused around the bookmarks.

\subsection{Session Lengths}

Session length is the total time a user accessed a video, regardless of the actions they may have taken whilst doing so. For example, a session may be longer than the actual length of the video if the user chose to rewatch segments, and/or pause.

Figures $7 \mathrm{a} \& 7 \mathrm{~b}$ show the CDF of both session and inter-seek times (discussion of inter-seek times follows in the next subsection). It can be observed from the session times that most users access each video for a very short time relative to its overall length (possibly just watching the events they are interested in). In particular, note that in the $\mathrm{arg}-\mathrm{scg}$ case around $80 \%$ of sessions lasted less than 15 minutes. Given that the video was 2.2 hours in length, 15 minutes corresponds to only $11 \%$ of the total video. A similar result was found with $e u$ rovision, with $80 \%$ of sessions lasting less than $12 \%$ of the total video duration. The average session duration was found to be only 11 minutes and 18 minutes for arg-scg and eurovision respectively.

We also found that a small minority (roughly 3\%) of session durations were longer than the length of a video. Of these durations roughly $39 \%$ were between 3 to 8 hours long. Our logs show that these users paused for a long time before deciding to resume playback. 


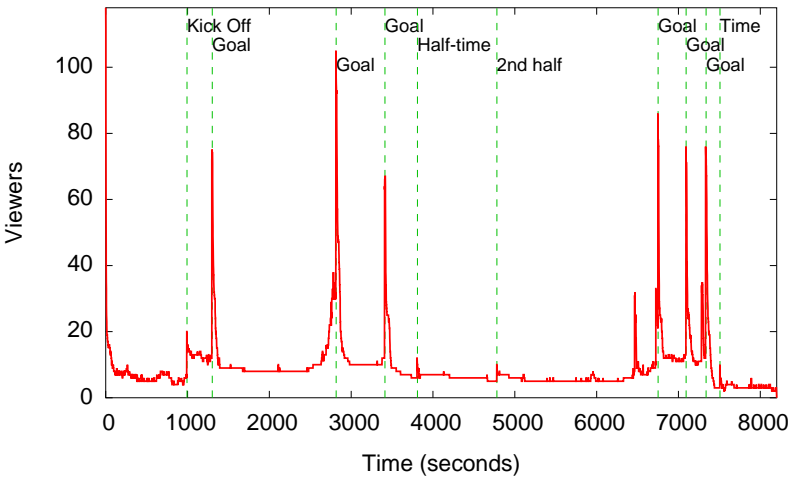

(a) Argentina vs. Serbia and Montenegro

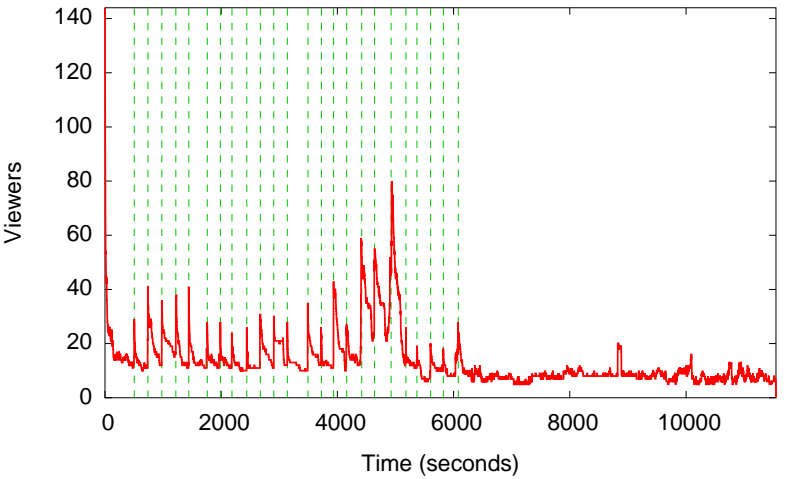

(b) Eurovision

Fig. 5: Number of viewers at each second of video (each vertical line represents the position of a bookmark)

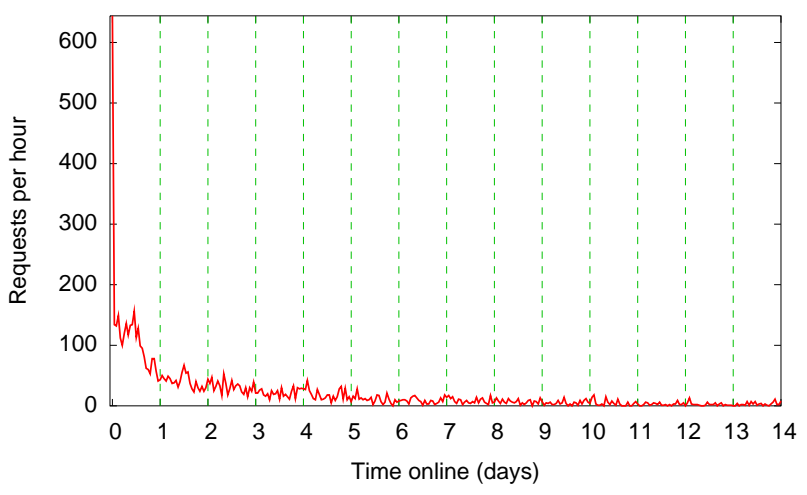

Fig. 6: Bookmark utilisation over time, following initial usage

\subsection{Inter-seek Times}

Inter-seek time is described as the duration for which a user watched a section of a video before seeking to a new location (disregarding any paused periods). This can be useful, for example, to determine the amount to replicate when using partial caching.

From our logs, we found that on average a user performed 8.98 seek operations around a video, resulting in a mean inter-seek time of 50.4 seconds. Figures $7 \mathrm{a} \& 7 \mathrm{~b}$ show the CDF for inter-seek times as well as session length. As the inter-seek times are generally shorter than session times, this implies that the majority of users viewed the content as a series of excerpts, usually under a minute in length.

The inter-seek time in the music content was found to be on average longer. This is because the length of a bookmarked musical performance generally exceeds the length of an event within a football match. Regardless of the difference in inter-seek times, we found that they can be estimated by log-normal distributions. For instance, the inter-seek time for arg-scg can be modelled with parameters $\mu=2.15$ and $\sigma=1.72$.

Previous studies have found that the majority of inter-seek times are very short [14]. For educational content, inter-seek times have also been shown to be Poisson or Pareto distributed [3]. We however found only two thirds of our videos had inter-seek times that could be suitably modelled by a Pareto distribution, and none that could be modelled well with a Poisson distribution. Models of inter-seek times can be used by a delivery system to determine the size of video replicas and the time available to react before a user seeks elsewhere in the video.

\subsection{Sequence}

The traces were analysed to study the extent to which users' actions could be predicted. Since jumps to bookmarks made up a relatively large percentage of all requests, we limit this prediction to which bookmark will be visited next. If a system could predict which bookmark would be requested next by a user, then it could pro-actively respond in order to optimise content delivery. For example, based on the next predicted bookmark, the relevant segments could be pushed out by a server with spare capacity, or pre-fetched by a client.

We call the order that bookmarks are viewed by a single user a sequence of bookmarks. Every user's sequence can be aggregated together to form a directed graph. Each node in the graph represents a bookmark with links between them representing the probability of seeking to that bookmark next. Figure 8 shows a section of one of these directed graphs depicted as a tree for clarity. The "Start" node represents the beginning 


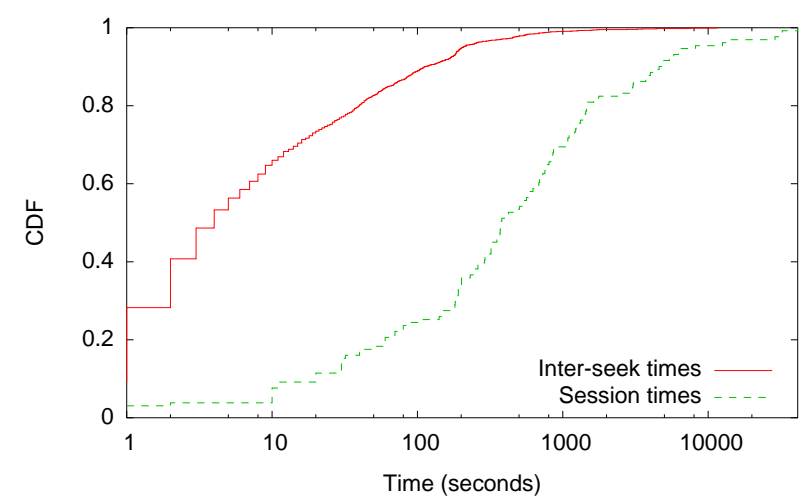

(a) Eurovision

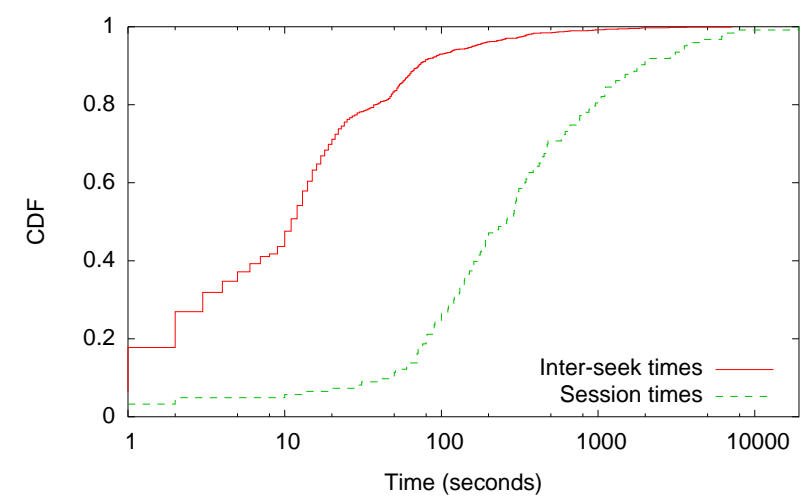

(b) Argentina vs. Serbia and Montenegro

Fig. 7: CDFs of session lengths and inter-seek times

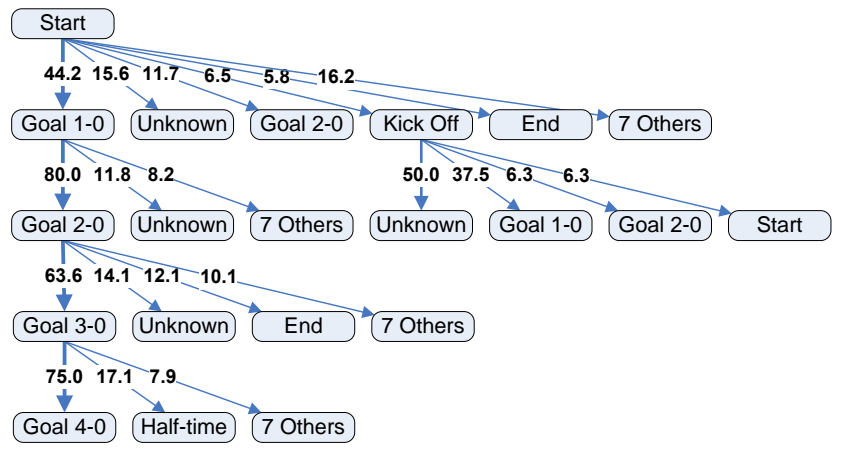

Fig. 8: Sequence diagram for Argentina vs. Serbia and Montenegro depicted as a tree

of the video, and the "End" node represents the completion of a session. There is also an "Unknown" node which signifies when a seek to another bookmark has not been made within 200 seconds of visiting the previous bookmark (the observed upper bound for bookmarked events' length). For clarity, links with low probabilities have also been aggregated to form a " $N$ Others" node, where $N$ is the number of aggregated links.

It is clear from the figure that there are multiple choices to visit from each node, although there is generally one link that is significantly more likely to be chosen. For example, the probability of viewing bookmark "Goal 2-0" immediately after "Goal 1-0" is $80 \%$. We can also see that following the "Kick Off" bookmark $50 \%$ of users did not visit another bookmark within 200 seconds and instead continue to watch, this could indicate that this subset of users were interested in watching the full game instead of just the highlights. An interesting observation for caching is the occurrence of self-loops. $6 \%$ of links were between the same two bookmarks, which made up $6.5 \%$ of all requests.

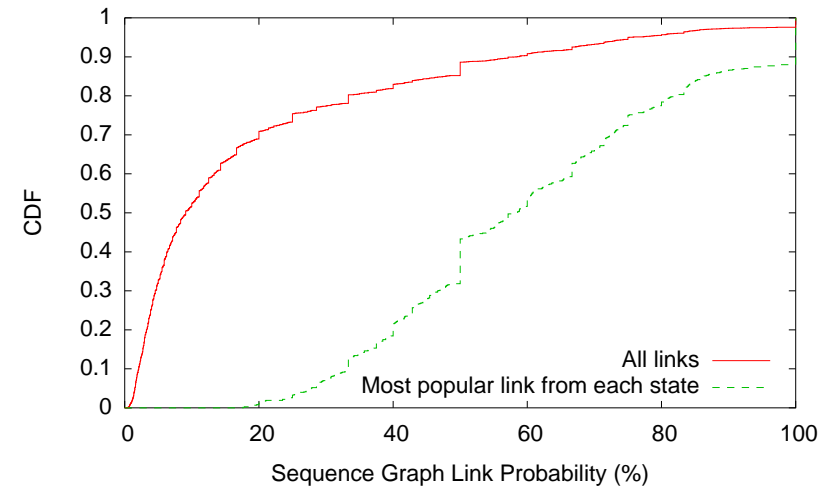

Fig. 9: CDF of link probabilities for all videos

To understand how many bookmark-to-bookmark links are predictable, Figure 9 shows a CDF of probabilities for all links for all videos, as well as probabilities for just the most popular link from each bookmark. From this figure we can conclude that $10 \%$ of all links have more than a $58 \%$ chance of being followed. Looking at just the most popular link from each bookmark we observe that over half of the bookmarks have an outgoing link with a probability over $50 \%$; an encouraging result for user predictability.

In this analysis we assumed that all users will visit the bookmark in similar order, however in a large heterogeneous environment this may not be true. Different sub-groups may wish to view a different set of events possibly in a different order to other sub-groups. Across our videos we did try and identify if there were groups of individuals that behaved differently to the majority, however none were found. This could possibly be due to our genre of media, with all sports fans wishing to see the same events, in the natural sequential order. 


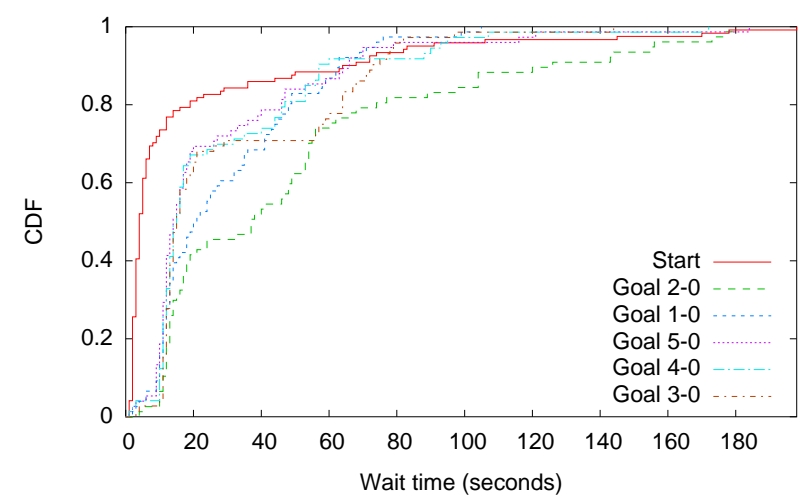

(a) Argentina vs. Serbia and Montenegro

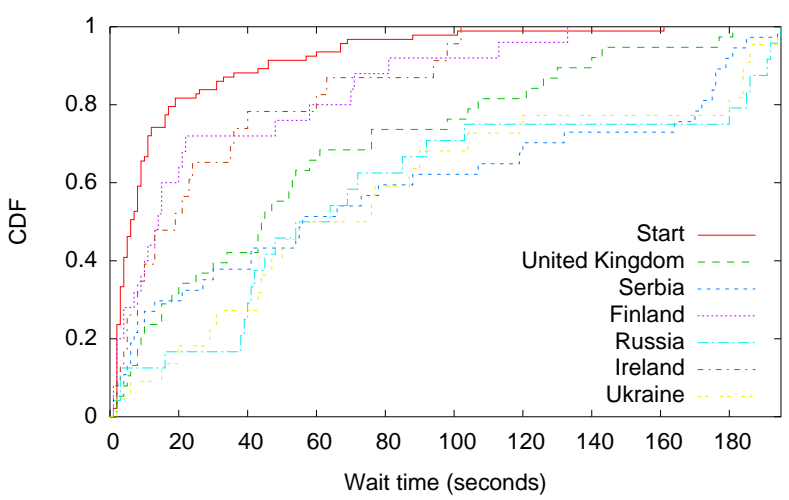

(b) Eurovision

Fig. 10: CDFs of wait times

\subsection{Hotspot Length}

Jumps to bookmarks comprised roughly $20 \%$ of all requests with an additional $32 \%$ of seeks being within 60 seconds of a bookmark. Bookmarks form the majority of requests within the content, and represent the beginning of a popular segment of video which we call a hotspot. The beginning of a hotspot is generally known (i.e., the bookmark point), but the end is not. Knowing the length of the hotspot can be useful for numerous tasks such as caching and pre-fetching. We therefore define wait time as the time elapsed between a user following a bookmark and seeking.

Figures $10 \mathrm{a} \& 10 \mathrm{~b}$ show a CDF of wait times for each bookmark in the arg-scg and eurovision videos. It can be seen that in the football match the wait times follow a similar distribution, with the majority of users waiting less than 40 seconds (this, for example, could corresponds to the length of a run up to a goal). The eurovision results are more varied with average wait times being much longer. This is due to the typical song in the Eurovision Song Contest being 180 seconds in length. Finally, there is a "Start" bookmark listed in both figures: this is the entry point into both videos, and does not correspond to any event.

To better understand the wait times, distributions were fitted. In the general aggregated case a Weibull model fits best with parameters $\lambda=24.594$ and $k=0.7034$. For individual bookmarks log-normal and Weibull models proved best in the majority of cases. With these models the upper bound of a hotspots' lengths can be extrapolated by using, for example, the $95^{\text {th }}$ percentile.

\subsection{User Behaviour Models}

Model fitting is important for understanding the different properties of the system, and aids in simulation creation and algorithmic design. Various models have been discussed for the different parameters of the system. In all cases many models (e.g., normal, log-normal, exponential, Weibull, Pareto, Poisson, Zipf) were fitted to the data with varying success. Generally, more than one distribution fitted well. This subsection will summarise the analytical models found for each parameter.

Table 2 gives an overview of the best matching models for each metric discussed previously, with their corresponding $R$-square values. Of particular importance are the types of distribution which can have a significant impact on the system. For example, the Weibull and log-normal models are both long-tailed, and systems may have to anticipate the skewed distribution to cope effectively.

The models shown so far are from aggregated results across all the videos. Instead, it may be interesting to model the different metrics of each particular video. However due to the diversity in models and parameters it is not possible to show each model, so instead Table 3 summaries which models fit with a $R$-square value greater than $95 \%$. The "max models" column represents the number of datasets that are of sufficient size to have models fitted. For example, there are 695 bookmarks, yet only 203 had enough data to be fitted to a hotspot length, and of these, 165 fitted well to a Log-normal model, 135 to a Weibull models, etc.

\subsection{Summary}

Our results have shown that the interactivity options available to users highly influence their behaviour. In 


\begin{tabular}{|c|c|c|}
\hline Metric & Distribution & R-square \\
\hline Object Popularity & Normal $(\mu=60.129, \sigma=32.111)$ & 0.97996 \\
Segment Popularity & Log-normal $(\mu=0.551, \sigma=1.32)$ & 0.98084 \\
& Weibull $(\lambda=2.887, k=0.69527)$ & 0.98284 \\
Session Length & Log-normal $(\mu=4.73, \sigma=1.90)$ & 0.99779 \\
& Weibull $(\lambda=233.17, k=0.51125)$ & 0.98666 \\
Inter-seek times & Log-normal $(\mu=1.2886, \sigma=2.318)$ & 0.99644 \\
& Weibull $(\lambda=7.5243, k=0.35646)$ & 0.99353 \\
Seek Distance (forward) & Log-normal $(\mu=7.2668, \sigma=1.2194)$ & 0.99567 \\
Seek Distance (backward) & Log-normal $(\mu=7.195, \sigma=1.3132)$ & 0.99083 \\
Hotspot Length & Log-normal $(\mu=2.6361, \sigma=1.388)$ & 0.98463 \\
Bookmark Longevity & Weibull $(\lambda=24.594, k=0.7034)$ & 0.99545 \\
& Weibull $(\lambda=3.1004, k=0.61592)$ & 0.99796 \\
\hline
\end{tabular}

Table 2: A summary of metrics with their corresponding distributions

\begin{tabular}{|c|c|c|c|c|c|c|c|c|}
\hline Metric & Max Models & Log-normal & Weibull & Pareto & Normal & Exponential & Zipf & No fit \\
\hline Segment Popularity & 84 (from 88 videos) & 61 & 65 & 12 & 58 & 42 & 13 & 17 \\
\hline Session Length & 81 (from 88 videos) & 75 & 72 & 0 & 5 & 31 & 4 & 0 \\
\hline Inter-seek times & 87 (from 88 videos) & 83 & 83 & 54 & 1 & 3 & 55 & 3 \\
\hline Hotspot Length & 203 (from 695 bookmarks) & 165 & 135 & 91 & 5 & 48 & 53 & 22 \\
\hline
\end{tabular}

Table 3: Metrics for individual videos and their corresponding distributions

particular, it was found that the novel interactive feature of bookmarking played a pivotal role, leading to access patterns quite dissimilar from previous related studies that looked at VCR-like interactivity alone. The combination of our content type and the addition of bookmarks led to users accessing content in relatively short segments sparsely distributed throughout the length of the videos. Segment popularity is skewed with the most popular segments clearly around the bookmarks, forming hotspots. From both a user and a CDN's perspective, this can be viewed as advantageous; users can reach interesting content more quickly through the bookmarks, and the increased locality of interest means CDNs can respond more effectively by, for example, prioritising hotspot replication.

Content placement is an important and difficult problem for CDNs. The CDN has to decide where within the network to replicate or cache content. Typically the content is placed near to the users, and replicated as a whole. However, as we have seen, not all segments within a piece of content are equal and a CDN can leverage this information to replicate certain segments more than others. This is especially useful when popularity nearly always concentrates around bookmarks, allowing the relevant segments to be replicated throughout the network before user demand increases.

A CDN could be designed to handle high levels of user interactivity, with relatively short sessions and inter-seek times. Our results have shown that hotspots following bookmarks were orders of magnitude shorter than the video containing them. Furthermore, it encourages the use of an agile delivery mechanism that al- lows distribution of small sparsely distributed segments quickly and efficiently.

We have also shown that users view the bookmarks in a similar order, giving them a degree of predictability. This could allow a CDN to exploit pre-fetching techniques to improve the user's experience. For example, if the CDN could predict the next segment the user will watch, then this could be pre-fetched into the user's playback buffer and when the user seeks to that segment there will be no delay caused by seek latency and buffering.

The use of bookmarks depends on them being well positioned and of interest to the user. We noted in the first experiment that $40 \%$ of bookmarks had at least one user seek before the bookmark, with $30.7 \%$ of these seeks occurring within 5 seconds of jumping to the bookmark. This perhaps represents users who were almost immediately dissatisfied with the bookmark's location. We noted this happened consistently for roughly $6 \%$ of the total bookmarks. Upon further inspection, it appeared the bookmarks were inadvertently misplaced. This led to users performing additional seeks to find the correct location, thus placing extra load on the servers.

In Section 5, we explore and study the implications of two techniques designed to exploit some of the properties suggested from our analysis.

\section{Techniques for Interactivity Support}

During our second video trial, we took the opportunity to go beyond characterising user behaviour, by testing autonomic content management techniques in a live system. In this section we discuss and analyse a simple 


\section{Scenario A}

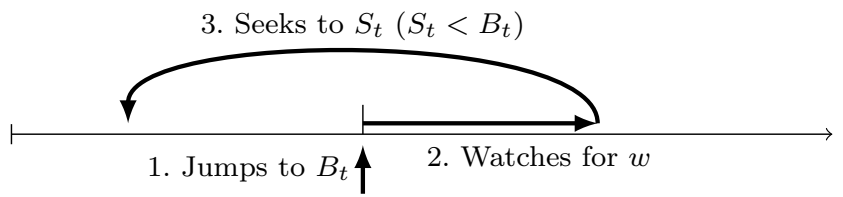

Scenario B

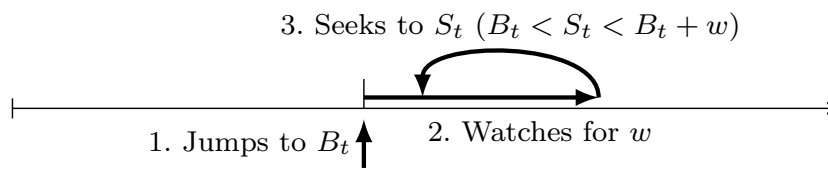

Scenario C

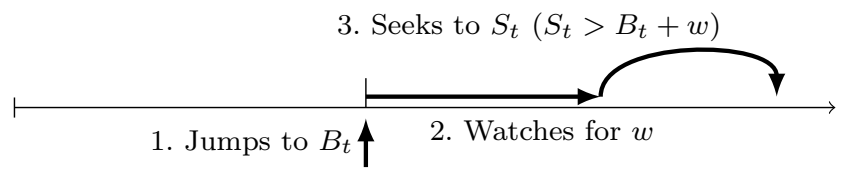

Fig. 11: Different scenarios that may induce bookmark movement

dynamic bookmark placement technique, as well as an interactivity-aware content pre-fetching method based on the prediction of which bookmark would be viewed next.

\subsection{Moving Bookmarks}

During our video trials, bookmarks were appropriately positioned by administrators before the video was published. It was previously noted that a small percentage of bookmarks were unintentionally misplaced. There are many reasons why a bookmark could be misplaced, such as human error, or a lack of insight into user requirements. For example: a bookmark could be placed before a penalty kick, but many users may first wish to see the foul that led to the penalty. As such, it could be beneficial if the system could autonomically detect poorly placed bookmarks and correct them based on feedback derived from the user's actions.

To develop a reactive algorithm that moves bookmarks dependent on user behaviour, different possible scenarios should first be explained. Figure 11 shows three different sequences of actions a user would follow shortly after seeking to a bookmark.

Scenario A shows the user briefly viewing the bookmark, then seeking to a time earlier than it. While this could indicate that the bookmarked event was short and that the user wanted to view it again, it could equally imply that the bookmark was placed later than it should have been.

Scenario $B$ is similar to Scenario $A$ but differs in that the user does not seek back to a point before the bookmark; this means the user is simply replaying footage, thus implying the bookmark is correctly placed for that individual.

Scenario $C$ represents a situation in which the user's motives are difficult to determine. Since they watch briefly then seek forward, several possibilities exist: the bookmarked event may have ended, the bookmark may have been placed prematurely, or the user is simply seeking forward towards the next event.

A further possibility, not shown in the figure, is for a user to seek far away from a bookmark in either direction. Since it is unlikely their destination would be related to the bookmark, such an action would not indicate the bookmark was incorrectly placed.

Scenario $A$ and Scenario $C$ are therefore the only scenarios where the user's actions could indicate the bookmark is misplaced. All other actions should reinforce the position of the bookmark to reduce future movements once it is correctly placed. Additionally since we are less sure of the user's intentions in Scenario $C$ we should only make minor changes to the bookmark's placement to limit the impact of false-positives.

Algorithm 1 has been developed to identify these situations and act appropriately with regard to moving a bookmark. An exponential moving average (EMA) is used to recalculate the bookmark's position with a smoothing constant $\alpha$. The value used for $\alpha$ is dependent on the identified scenario. Initially these values were 0.1 and 0.05 allowing us to place greater confidence in the seeking-backward Scenario $A$ than the seeking-forward Scenario $C$. These values were chosen as the intuitive first guesses for experimental purposes, and should be refined with future experiments. For our testing scenario we also used maximum wait times of 20 and 60 seconds for backward and forward seeks respectively. These maximum values were chosen because they exceeded approximately $80 \%$ of all wait times.

To test this algorithm, several of the bookmarks in our second video trial (not our initial World Cup experiment) were deliberately misplaced by different amounts before they appeared on the live site. Over time the bookmarks were moved autonomically by our algorithm. For example, Figures $12 \mathrm{a} \& 12 \mathrm{~b}$ show the position of a single bookmark as it was moved by the system with respect to time and received requests. In both cases the system responds and the bookmark quickly moves to a new position, and then gradually converges until it becomes stable. In most cases the majority of movements were only in one direction, but for a cou- 


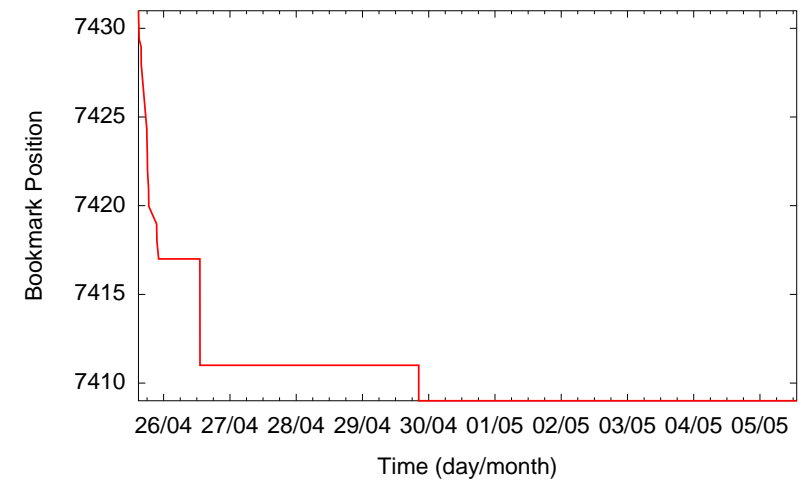

(a) Position over time

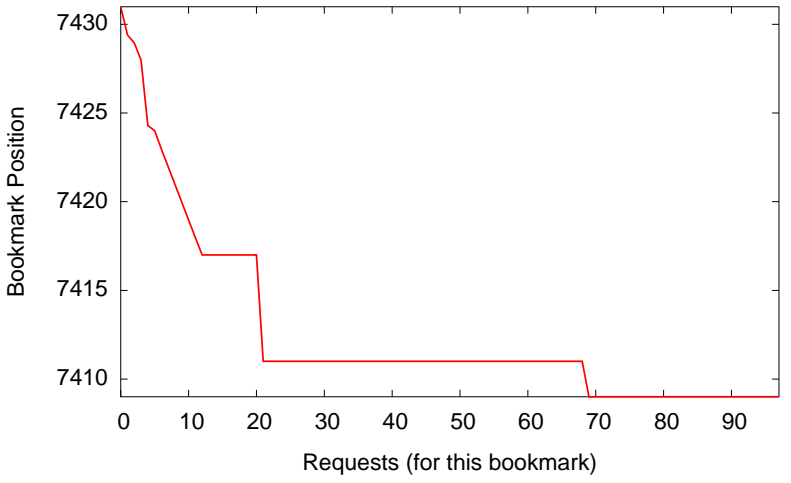

(b) Position over requests for bookmarks

Fig. 12: Manchester United vs Milan single bookmark position

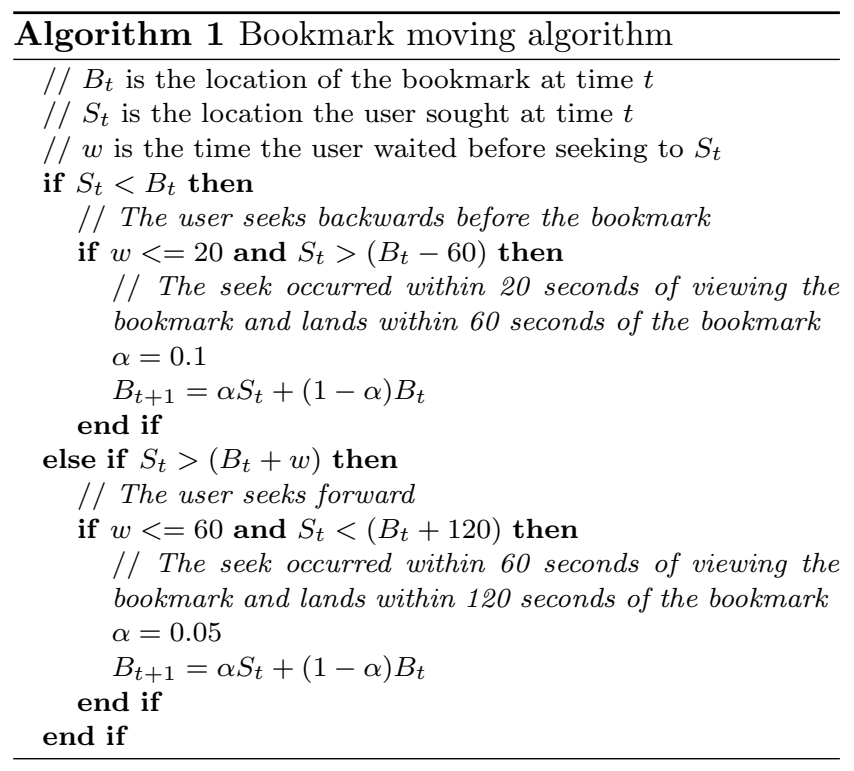

ple of bookmarks the positions oscillated between two values. The most prominent example of this was a foul in a football match which led to a penalty. Some users wished to see the foul but others only wished to see the penalty a minute later. In these small number of cases it is subjective to decide if a bookmark is correctly placed, and in fact using this algorithm the bookmarks may never converge to a single point. In such cases, it may be best to bias the bookmark towards the earlier position, so both the early and later events can easily be seen.

Instead of subjectively deciding if a bookmark has moved to its correct location, we examined how much traffic might have been saved by moving the bookmark to a new location. If, for example, a bookmark was moved forward 10 seconds closer to the desired location, and a user views for 90 seconds, then by moving the bookmark we have potentially stopped video being transferred which might have normally been skipped over. A reduction of $10 /(90+10)=10 \%$ is therefore made. Of course, this is only true if the user does not seek backward to watch the skipped 10 seconds, in which case we save nothing, and in fact incur an extra seek. Figure 13a displays a CDF of the potential reduction in viewing duration per bookmark request from the use of the algorithm. We can see that $16 \%$ of the requests made no saving: these are accounted for by early requests before the bookmarks were moved, and requests where the user incurs an additional seek.

Figure 13b illustrates how rapidly these reductions are made (and whether or not they are sustained) through a plot of the fractional potential saving versus the number of requests received across all the moved bookmarks. For the first $20 \%$ of requests the reductions are low yet they improve, and then stabilise at a reduction of between 30-40\% per request. The $95 \%$ confidence intervals are quite wide in most cases (averaging around \pm 10 seconds) although this variance is mostly due to differences in playback length and not the $16 \%$ of requests with no saving.

With minimal processing this simple algorithm has been able to reposition the bookmarks to more appropriate locations based on observed user behaviour, resulting in consistent traffic reductions. The algorithm can still be improved by fine tuning the $\alpha$ values. Larger values would move the bookmark more quickly at the cost of increasing the probability of incorrect decisions. This investigation has been left for future work.

\subsection{Predictive Pre-fetching}

Due to the increased interactivity of users and their departure from the start-to-finish model, it is no longer 


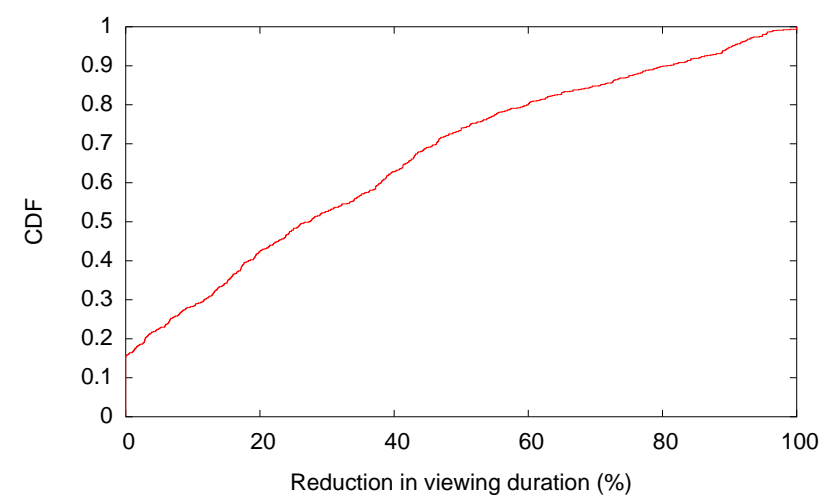

(a) $\mathrm{CDF}$ of percentage reduction in viewing duration

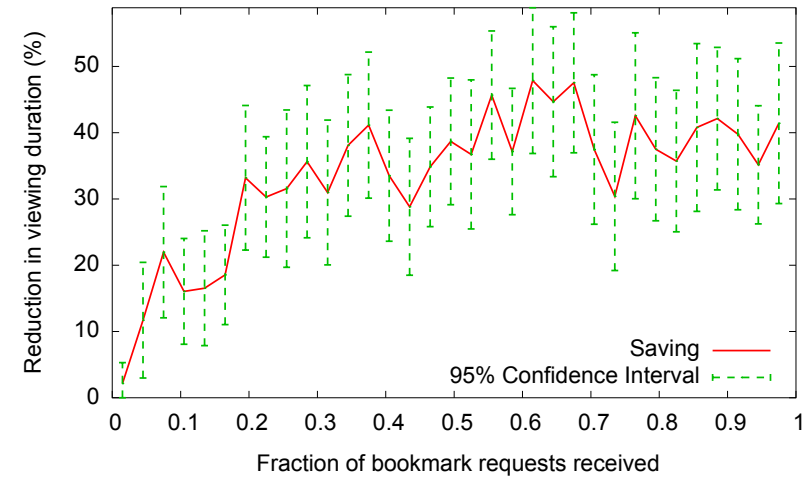

(b) Percentage reduction in viewing duration versus received requests

Fig. 13: Reduction in viewing duration due to the algorithm

wise to only pre-fetch ahead of the playback point. However, as noted in Section 4.7 it is still possible to predict which bookmark a user will view next, allowing the client to intelligently pre-fetch content, benefitting both clients and servers. For the clients, pre-fetching removes seek latency when seeking to a pre-fetched segment, both in terms of the network seek latency incurred and also the time taken to buffer enough video for playback, as well as helping to avoid buffer underruns under poor network conditions. Similarly, on the server side, pre-fetching can help reduce the peak server load by increasing the load at quieter times with prefetching requests, thus making the overall load more uniform.

However, pre-fetching does come with a cost; resources are wasted if a segment is downloaded and never used. Deciding which segments to pre-fetch is therefore an important task. We devised a set of pre-fetching strategies which we tested within a simulator driven by the eurovision trace obtained from our system. Clients were provisioned with a dedicated link to the server, capable of transferring twice the bitrate required to play the content. Once a client has fetched enough data to fill a 5 second playback buffer, half of their bandwidth is allocated to the pre-fetcher whilst the other half continues to fill the playback buffer.

For simplicity, and because interest always formed around bookmarks, each strategy will only pre-fetch segments immediately following a bookmark (i.e., bookmark hotspots). In all experiments the amount of each hotspot pre-fetched was determined by varying the percentile of that particular hotspot's length model, as described in Section 4.8. The details for each pre-fetch strategy are listed below:
Ahead simply continues to pre-fetch ahead of the playback point assuming the client has a unlimited buffer. This is similar to what some existing streaming applications do.

Ahead (to hotspot end) again simply continues to pre-fetch ahead of the playback point but only until the end of hotspot associated with the bookmark being viewed.

Ahead (and Predictive) works in a similar way to Ahead (to hotspot end), however once it reaches the end of the hotspot it begins to use the Predictive prefetch scheme.

Predictive uses knowledge observed from other users as to which bookmark is likely to be requested next, and thus starts to pre-fetch the bookmark hotspots in descending order of probability of being visited. The more users interacting with the system, the more accurate the predictive knowledge becomes.

Sequence will pre-fetch bookmark hotspots in the order in which they appear within the video regardless of the current playback point. For example, in a football match the goals would be pre-fetched in a sequential order.

Sequence After again pre-fetches bookmark hotspots in the order in which they appear within the video; the difference being only hotspots that are after the current playback point are fetched. For example, if a user has yet to fetch the first bookmark's hotspot but is already viewing the second, then the first will not be pre-fetched.

Two metrics were measured to determine how well the different schemes behaved. The first metric displayed in Figure 14a is the fraction of requests with zero seek latency. A seek latency of zero occurs when the user has already pre-fetched a playback buffer's worth 


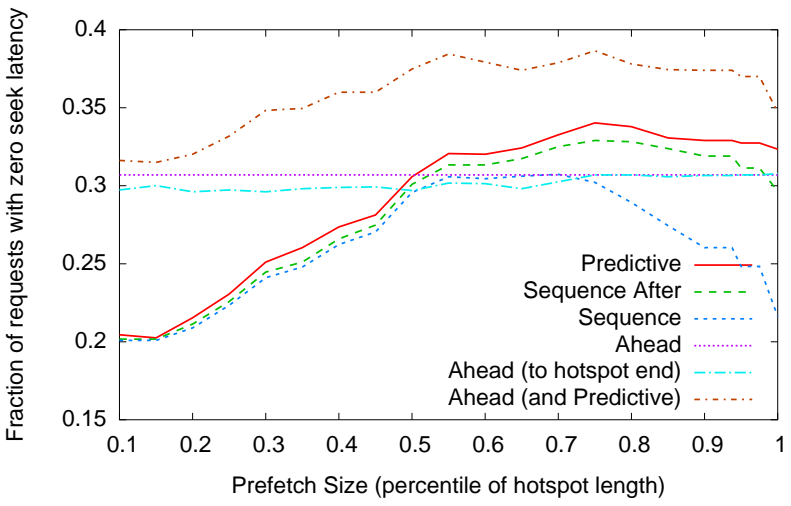

(a) Fraction of requests with zero seek latency

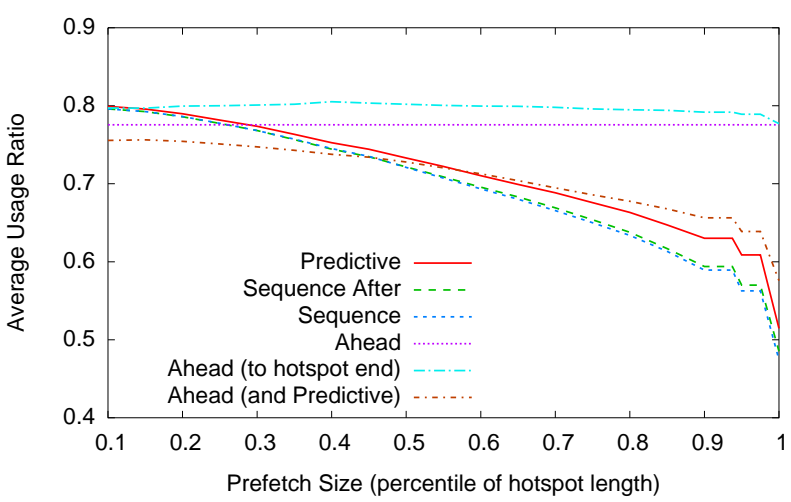

(b) Usage ratio

Fig. 14: Various metrics for different pre-fetch schemes versus bookmark length

of video from a requested seek point. The second metric measured the ratio of fetched data which was never watched, and therefore needlessly fetched. This usage ratio is shown in Figure 14b.

Using the simple Ahead scheme $31 \%$ of seeks have zero latency, this is made up of seeks to segments that have already been viewed, and small forward seeks into the ahead buffer. Adapting this scheme to only prefetch to the end of the bookmarks (i.e. the Ahead (to hotspot end) scheme) has a minor negative effect on the seek latency, whilst increasing the average usage ratio.

The Sequence and Sequence After schemes are very similar, but the simple modification to the Sequence After scheme allowed it to achieve a lower seek latency whilst not degrading its average usage ratio. This was because users had a tendency to not seek to a bookmark before the current playback point, and always go forward within the video, leaving the Sequence scheme stuck pre-fetching hotspots before the current playback point.

Both the Predictive and the Sequence After schemes perform in a similar manner, with the Predictive schemes always outperforming the other. Due to this fact, the Sequence After scheme could be used in place of the Predictive scheme whilst knowledge is collected to improve the Predictive scheme's accuracy.

The best outcome was the combination of Ahead and Predictive schemes named Ahead (and Predictive). This exploited the fact that users rarely viewed beyond the end of a hotspot, and thus pre-fetching another hotspot was of benefit.

In the previous experiments the Predictive scheme was primed with knowledge from all users, but in reality this knowledge would be built up over time. To test how quickly this knowledge can be obtained we ran another set of experiments where the Predictive and Ahead (and

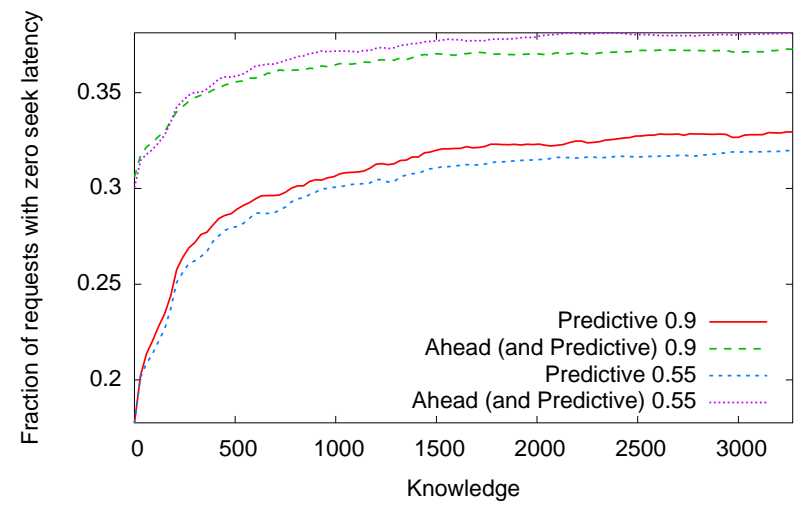

Fig. 15: How zero seek latencies is effected by amount of pre-fetch knowledge

Predictive) schemes were primed with different amounts of knowledge. The results of this are shown in Figure 15. We set the percentile hotspot length to 0.55 and 0.9 which were chosen since 0.55 is where the seek latency began to stabilise, and 0.9 where the usage ratio began to drop rapidly. The knowledge is ranked from 0 to 3000 which represents the number of seek requests received. It can be seen that very quickly (within 250 seek requests) the knowledge has become useful, and eventually plateaus at 1500 seek requests. Any seek requests after this point just increase the confidence in the knowledge and does not improve it.

\section{Conclusions and Future Work}

We have presented a study and characterisation of user behaviour for our interactive Video-on-Demand system. We note that by adding simple bookmarks to points of interest within the media, the access patterns are greatly influenced. This behaviour led to high levels 
of seeking which created relatively-short and sparselydistributed segments with orders of magnitude more popularity than others.

Various distributions were fitted to the different metrics considered, providing a greater understanding of how users interact with such systems. Beyond the insight gained, the models constitute a valuable toolbox for driving future simulations which require highly interactive workloads.

Many existing delivery mechanisms are not designed for high levels of interactive behaviour and are instead optimised for classic start-to-finish streaming. CDNs must therefore adapt to efficiently handle these kinds of access patterns. They could, for example, take advantage of the long-tailed distributions of segment popularity by replicating those that generate the most demand. For instance, we observed that $10 \%$ of segments accounted for $44 \%$ of all requests.

The departure from classic start-to-finish playback encourages the design of agile delivery mechanisms that allow quick seeking, and allow certain more popular segments to be more highly distributed. We have seen that adding bookmarks will highly influence the order in which users view the content, making the sequence of actions somewhat predictable. This can then be exploited by allowing users to pre-fetch content that they are predicted to need shortly, thus reducing any delays they are likely to experience. However, we noted that bookmarks could be harmful by causing unnecessary seeks if incorrectly placed. This could be remedied for both client and server by simply moving the bookmark autonomically based on observed user behaviour.

So far we have only considered bookmarks within music and sport videos, but bookmarks are equally applicable in many other genres. For example, bookmarks are commonly found in the form of chapters on video DVDs. It is not clear if the same high levels of interactivity would be observed, or if the classic start-to-finish model would still be prevalent.

For a system to be fully autonomic the bookmarks should perhaps be created automatically. This could occur after the system has detected a large number of requests for a specific area of a video. A bookmark could then be provisionally placed and its position refined by a bookmark-moving algorithm, such as the one found in Section 5.1.

During our experiment users were unhappy that we "spoilt the experience" of watching the sporting events covered somewhat. This was because the user could quickly determine the final outcome of the event from the bookmark names. The suggestion was made that we avoid labelling the bookmarks and instead simply describe them as points of interest. This could equally work if the bookmarks were autonomically created since a system would be unable to name them itself. Note, unnamed bookmarks would only be useful if they are typically accessed sequentially, and not based on their name alone.

It was clear that pre-fetching bookmark hotspots only covered $35 \%$ of all viewed segments. Thus prefetching schemes should consider more segments. This of course would make it harder to decide which segments to pre-fetch next. The probability of making a wrong decision could be reduced if the pre-fetching technique was modified, for example pre-fetching more than one choice simultaneously.

The predictive pre-fetching algorithms used knowledge inferred from the observations of other users. For example, if the majority of all users visited two bookmarks in the same order, it is likely the next user will do the same. How this knowledge is collected, and how this knowledge is disseminated is left open for future study. We did not discuss who would use this knowledge, indeed both the client and server could exploit it differently, each with respective pros and cons. For example, if the client has spare capacity, it could start to pre-fetch based on both its own previous behaviour and that of the majority of other users. The server could also decide to pre-replicate, or to push out segments predicted to be required when it has spare capacity.

While not the case for all content, high levels of interactivity are becoming more common, whilst users are both relying on and expecting Video-on-Demand services to provide more advanced interactive functionality. Our study suggests that CDN mechanisms must improve to handle more diverse applications, content and users. To achieve this, the development of new algorithms must be driven by models derived from realistic characterised workloads. The development of such strategies is reliant on gaining a deep understanding of the relevant workload parameters. The analysis and models presented in this paper aim to aid in this endeavour.

\section{References}

1. S. Acharya, B. Smith, and P. Parnes. Characterizing user access to videos on the world wide web. In Proceedings of Multimedia Computing and Networking (MMCN), San Jose, California, USA, January 2000.

2. Akamai. Content distribution network. http://www.akamai.com.

3. J. Almeida, J. Krueger, D. Eager, and M. Vernon. Analysis of educational media server workloads. In Proceedings of the 11th International Workshop on Network and Operating Systems Support for Digital Audio and Video (NOSSDAV), Port Jefferson, New York, USA, June 2001. 
4. S. Chen, B. Shen, S. Wee, and X. Zhang. Adaptive and lazy segmentation based proxy caching for streaming media delivery. In Proceedings of the 13th International Workshop on Network and Operating Systems Support for Digital Audio and Video (NOSSDAV), pages 22-31, 2003.

5. L. Cherkasova and M. Gupta. Characterizing locality, evolution, and life span of accesses in enterprise media server workloads. In Proceedings of the 12th International Workshop on Network and Operating Systems Support for Digital Audio and Video (NOSSDAV), Miami Beach, Florida, USA, May 2002.

6. M. Chesire, A. Wolman, G. M. Voelker, and H. M. Levy. Measurement and analysis of a streaming media workload. In Proceedings of the 3rd USENIX Symposium on Internet Technologies and Systems, San Francisco, California, USA, March 2001.

7. C. Costa, I. Cunha, A. Borges, C. Ramos, M. Rocha, J. Almeida, and B. Ribeiro-Neto. Analyzing client interactivity in streaming media. In Proceedings of the 13th International World Wide Web Conference ( $W W W$ ), New York, New York, USA, May 2004.

8. P. Danzig, J. Mogul, V. Paxson, and M. Schwartz. Internet Traffic Archive. http://ita.ee.lbl.gov.

9. G. S. Fishman and I. J. B. F. Adan. How heavytailed distributions affect simulation-generated time averages. ACM Transactions on Modeling and Computer Simulation (TOMACS), 16(2):152-173.

10. L. Guo, S. Chen, Z. Xiao, and X. Zhang. Analysis of multimedia workloads with implications for Internet streaming. In Proceedings of the 14th International World Wide Web Conference $(W W W)$, Chiba, Japan, May 2005.

11. M. Mitzenmacher. A brief history of generative models for power law and lognormal distributions. Internet Mathematics, 1(2):226-251.

12. K. Sripanidkulchai, B. Maggs, and H. Zhang. An analysis of live streaming workloads on the Internet. In Proceedings of the ACM SIGCOMM Internet Measurement Conference $(I M C)$, Sicily, Italy, October 2004.

13. W. Tang, Y. Fu, L. Cherkasova, and A. Vahdat. MediSyn: A synthetic streaming media service workload generator. In Proceedings of the 13th International Workshop on Network and Operating Systems Support for Digital Audio and Video (NOSSDAV), Monterey, California, USA, June 2003.

14. M. Vilas, X. Paneda, R. Garcia, D. Melendi, and V. Garcia. User Behaviour Analysis of a Video-On-Demand Service with a Wide Variety of Subjects and Lengths. In Proceedings of the 31st EUROMICRO Conference on Software Engineering and Advanced Applications, pages 330-337, 2005.

15. H. Yu, D. Zheng, B. Zhao, and W. Zheng. Understanding user behavior in large scale video-on-demand systems. In Proceddings of EuroSys, 2006. 